\title{
Estimation of Eastern Denmark's Electromechanical Modes from Ambient Phasor Measurement Data
}

\author{
Luigi Vanfretti, Rodrigo García-Valle, Kjetil Uhlen, Emil Johansson, Daniel Trudnowski, \\ John W. Pierre, Joe H. Chow, Olof Samuelsson, Jacob Østergaard, and Kenneth E. Martin
}

\begin{abstract}
In this paper we report on the preliminary results of a collaborative investigation effort between researchers in North America and Europe aiming to baseline the electromechanical modes and mode shapes of the Nordic system from synchronized phasor measurement data. We provide an overview on the Danish power grid and its interconnections, and describe the features of an experimental phasor measurement unit designed by DTU which is used to provide the measurements used in this investigation. Parametric and non-parametric block processing techniques are applied to $\mathbf{4 8}$ hours of ambient data recorded at the Radsted and Hovegård substations in Eastern Denmark. The estimated modes are in agreement with those shown in eigenanalysis studies, and other measurement-based investigations. More importantly, the emergence of a new $0.8 \mathrm{~Hz}$ mode in Sjælland is reported.
\end{abstract}

Index Terms-synchronized phasor measurements, power system identification, power system monitoring, power system parameter estimation

\section{INTRODUCTION}

$\mathbf{S}$ PECTRAL analysis techniques have been successfully applied to characterize the small signal oscillatory modes in the US WECC interconnection [1], [2] and more recently in the US Eastern Interconnection [3]. In this paper we report on the preliminary results of a collaborative research effort between researchers in North America and Europe aiming to baseline the electromechanical modes and mode shapes as observed from PMU data recorded in the Nordic system. First, we provide an overview on the Danish power grid and its interconnections to Germany and the rest of the Nordic System. In addition, we briefly describe some of the features of an experimental phasor measurement unit designed by DTU which is used to provide the measurements used in this investigation.

Ambient data analysis is used to estimate the inherent oscillatory modes of the power system when the main source of excitation of the system modes are random load variations

L. Vanfretti and J. H. Chow are with the Department of Electrical, Computer, and Systems Engineering, Rensselaer Polytechnic Institute, Troy, NY 12180, USA (e-mail: lvanfretti@ieee.org, chowj@rpi.edu)

R. García-Valle and J. Østergaard are with the Department of Electrica Engineering, Technical University of Denmark, Lyngby, Denmark (e-mail: rgv@elektro.dtu.dk, joe@elektro.dtu.dk)

K. Uhlen and E. Johansson are with Sintef, Trondheim, Norway, (e-mail: kjetil.uhlen@sintef.no,Emil.Johansson@sintef.no)

Daniel Trudnowski is with Montana Tech, The University of Montana, Butte, MT 59701 USA (e-mail: dtrudnowski@mtech.edu).

J. W. Pierre is with the University of Wyoming, Laramie, WY 82071 USA (e-mail: pierre@uwyo.edu)

O. Samuelsson is with the Department of Measurement Technology and Industrial Electrical Engineering, Lund University, Lund, Sweden (email:olof.samuelsson@iea.lth.se)

K.E. Martin is with the Electric Power Group, Pasadena, CA, USA. (e-mail: martin@electricpowergroup.com) resulting in a low amplitude stochastic time series referred to as "ambient noise" [4]. Non-parametric and parametric techniques, such as the Welch periodogram [5], [6], [7] and Yule-Walker method [8], [2], can be used to determine system modes, which are visible peaks in the spectrum estimate. Spectral estimates may also be used for mode shape estimation, the cross spectral function (CSD) can be used to estimate the phasing of the mode among the system generators, and coherency can be determined by the squared coherency function [9], [10]. Because these algorithms rely on block processing of data windows, they require several minutes of time-synchronized phasor data from different locations in the power network. This study focuses on the application of block processing spectral techniques for mode estimation only.

The estimated interarea modes obtained in this paper are in agreement with other investigations using measurements [11], [12] and studies that use eigenanalysis techniques applied to planning models [13]. The results in this paper are of value to power planners in the Nordic system as they may serve to refine their power system models. In addition, we report on the discovery of a $0.8 \mathrm{~Hz}$ oscillatory mode in Eastern Denmark that has not been discussed in previous investigations. This mode is likely to be of interarea nature involving generators in Sweden and Eastern Denmark.

\section{ThE DTU-PMU AND ITS DEPLOYMENT IN THE DANISH GRID}

\section{A. The Danish Electric Power System}

The Danish power system is divided in two parts, Eastern and Western. Each of these parts belong to different synchronous AC systems. The Eastern Danish system belongs to the Nordic system (previously Nordel), while the Western system belongs to the continental European system (previously UCTE (Union for the Coordination of Transmission of Electricity)).

The power systems in Eastern and Western Denmark have several important differences. On Sjælland the $132 \mathrm{kV}$ transmission network consists of ring connections, while the $400 \mathrm{kV}$ grid is characterized by a radial structure.

The transmission grid in Eastern Denmark comprises overhead transmission lines and underground cables at the two highest voltage levels of $132 \mathrm{kV}$ and $400 \mathrm{kV}$, and the interconnections with South Sweden and Germany.

In Jylland and Fyn, the $400 \mathrm{kV}$ network is a combination of ring connections and radial structure. The $150 \mathrm{kV}$ grid is a parallel network which also plays a role in connection with the international transmission capacity market. 


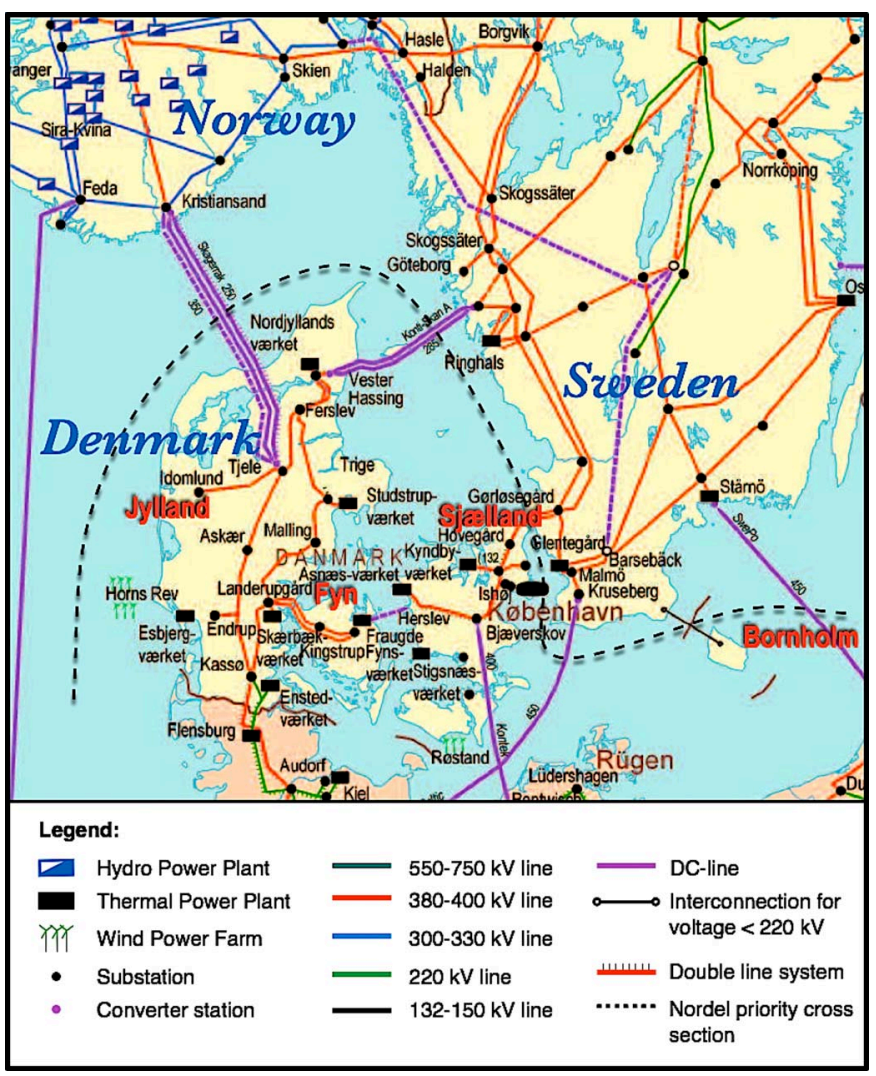

Figure 1. The Danish power grid and its interconnections

The electric power transmission system in Denmark has always been divided by the Great Belt between Sjælland and Fyn, but there are plans to connect the two transmission systems with a DC cable.

\section{B. The DTU-PMU}

This unique phasor measurement device consists of two PCunits that combine to perform a PMU function. One PC unit runs only the disk operating system (DOS). It has an interrupt system designed for reasonable handling of holds from administrative peripherals, such as timer, keyboard, USARTS (Universal Synchronous/Asynchronous Receiver/Transmitter), direct memory access (DMA), etc. This can be altered by the user so that the user's own processes can have priority operation. The second system has a Windows 2000 operating system which facilitates higher level administrative tasks, such as data $\mathrm{I} / \mathrm{O}$, file creation and storage, etc.

To obtain accurate phasor estimates, the PMU estimates the frequency of the AC power input signal and synchronizes sampling with it. Synchronizing the sampling to the actual power system signal eliminates problems in phasor estimation. 64 or 128 samples over the fundamental frequency period are taken with a 16-bit A/D converter. The phasor is calculated with a Goertzel algorithm, which is a simplified FFT analysis that only extracts one frequency component. The next task is translating the phasor to the UTC time reference.

In addition to keeping an internal frequency estimate, the PMU also synchronizes an internal clock with the external UTC time derived from GPS. It does this with a software

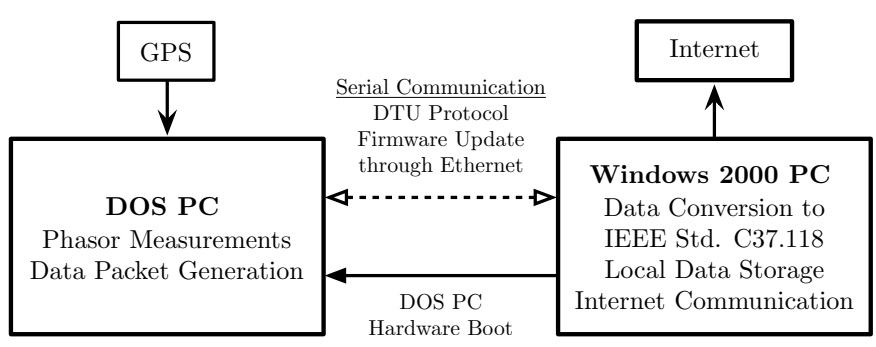

Figure 2. The DTU PC-Based Phasor Measurement Unit

clock that is synchronized to the GPS receiver. The GPS will latch and report a time stamp based on a signal from the PMU. This allows the PC to synchronize its internal time clock within $1 \mu$ s of UTC time, based on the GPS accuracy of \pm 100 ns. Synchrophasor reporting is based on UTC time intervals. After each phasor is calculated, the PMU compares the phasor estimate time with the UTC time. If it is time to report a synchrophasor, the PMU estimates the UTC referenced phase angle and magnitude from the 1 or 2 phasors proportionately that cover the interval. Note that the calculated phasors are synchronized with the power system and will probably not coincide UTC reporting intervals. The equivalent synchrophasor must be estimated from 1 or 2 phasors and the angle must be adjusted to the UTC time.

The DOS PC will report the estimated synchrophasor to the windows PC based instrument. The production unit can locally store up to eight phasors for every UTC $20 \mathrm{~ms}$ time window. All data is stored locally; there is no real-time data output in this version. Data files can be remotely downloaded without interrupting data acquisition and storage. The main characteristics of the DTU-PMU are:

- 16 channels sampling at a rate of $1600 \mathrm{~Hz}$

- 300 ns time stamp for each sample

- FFT analysis for $50 \mathrm{~Hz}$ component

- Phase accuracy of $\approx 0.5 \mu$ s $(\approx 0.01$ degree $)$

- Data collection through the Internet

- Uninterrupted local data storage on the PC's hardrive

- Transmission of one data package for each $20 \mathrm{~ms}$ in UTC time

\section{DTU PMUs in the the Danish Grid}

Ten PMU devices were manufactured and placed in different locations around the Danish power grid using the phasor measurement technology discussed above. Figure 3 shows the location of the DT-PMUs installed in in Sjælland. The distribution of the PMUs is as follows:

(1) One PMU at Snæsværket $(400 \mathrm{kV})$

(2) Two PMU in Hovegård (400 and 132 kV)

(3) One PMU in Radsted $(132 \mathrm{kV})$

(4) One PMU in Nysted (165 MW offshore windfarm)

(5) One at the CET-DTU $(0.4 \mathrm{kV})$ - not shown in Fig. 3

\section{Mode Estimation from Ambient Phasor Data}

Ambient data analysis is used to estimate the inherent oscillatory modes of the power system when the main source 


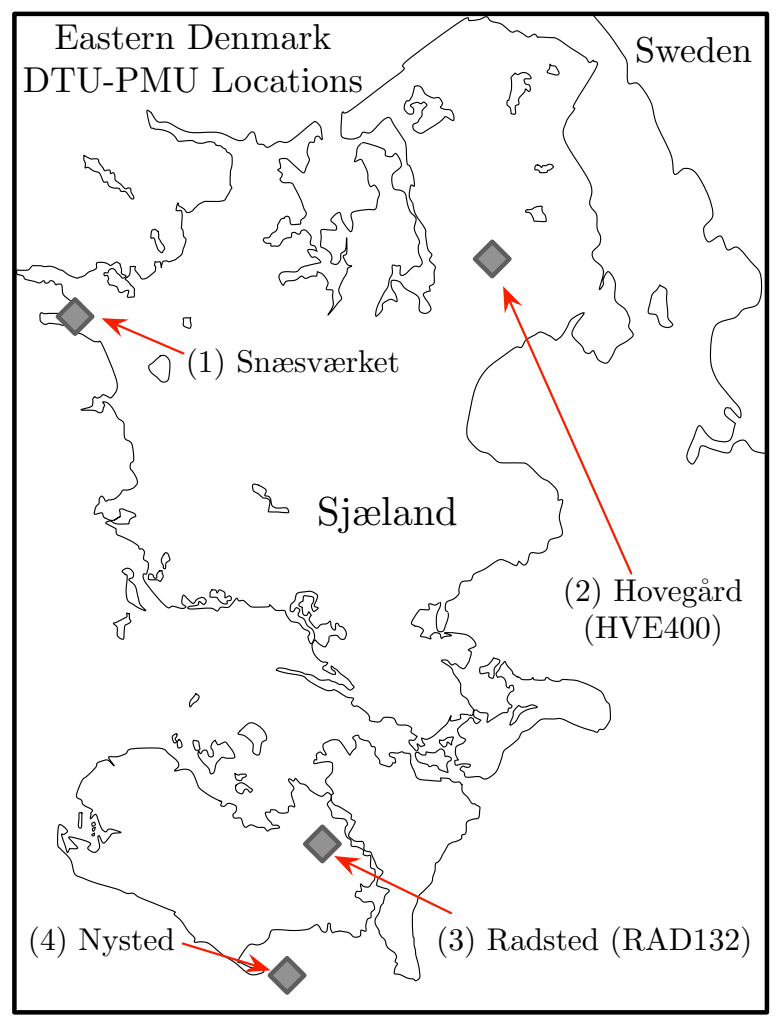

Figure 3. Deployment of the DTU-PMU in Eastern Denmark

of excitation of the system modes are random load variations resulting in a low amplitude stochastic time series referred to as "ambient noise" [4]. There is a significant array of different methods available to perform ambient data analysis [4]. Here, we limit the discussion to block processing non-parametric and parametric methods. Block processing algorithms can determine mode estimates from a window of data, each window providing a new estimate. Therefore, these methods require a large amount of phasor measurement data, and may not be suitable for real-time applications.

A specially robust non-parametric spectral estimation method is the Welch periodogram [5], [6], [7], which gives an estimate of a signal's strength as a function of the frequency. Here the dominant modes will be shown as significant peaks in the spectral estimate. This method is very insightful and uses limited assumptions, however, numerical estimates of the damping ratio and mode frequency are not directly provided. The most popular parametric method is the Yule-Walker algorithm [8], [2] which is used to estimate the system modes using an autoregressive-moving-average (ARMA) model. Several variations of this method have been proposed in the literature [4].

In this study we have used parametric and non-parametric algorithms, the Yule-Walker and Welch algorithms, respectively; to obtain spectrogram estimates of the active power flow measurements and bus frequency at the two PMU locations. To obtain damping ratio estimates we use an energy technique implemented within a mode meter algorithm similar to [2], [4]. As a comprehensive discussion of the methods is beyond the scope of this paper, the reader is referred to the cited references for further details.

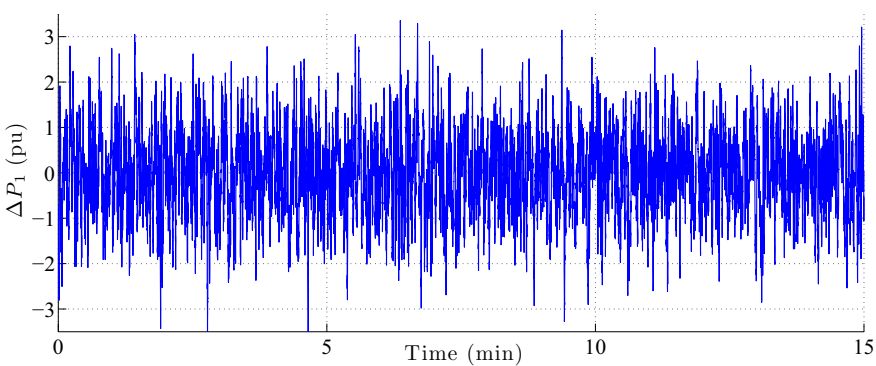

(a) Sample detreded 15 min. data block

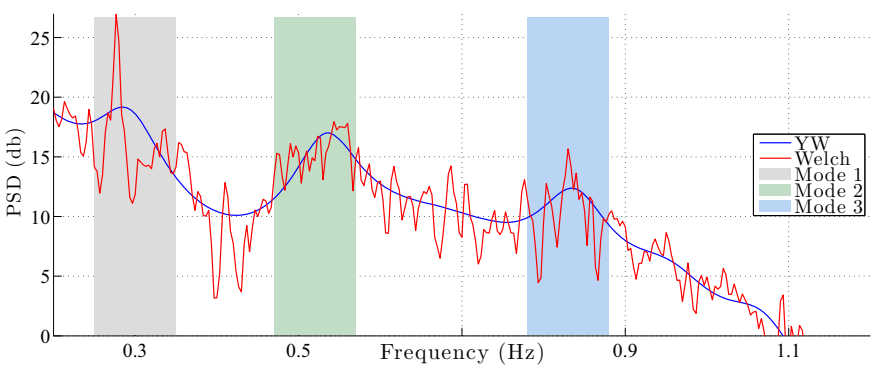

(b) PSDs from the Welch and Yule-Walker Methods

Figure 4. A detrended $15 \mathrm{~min}$. data block of the $P_{1}$ signal of Radsted, and its estimated PSDs using the Welch and Yule-Walker methods

\section{A. PSDs for Radsted and Hovegård}

For this investigation, we have used ambient data recorded for a period of 48 hours on two different dates and locations. Data from the Radsted substation (RAD132, in Fig. 3) were obtained during 03-20-2008 and 03-21-2008. The data for the Hovegaård substation (HVE400, in Fig. 3) was obtained during 04-15-2007 and 04-16-2007.

In several signals available from the PMUs the modal content was not strong enough, and thus we have not included any of their corresponding results here. For the active power flow, the low modal content is due to the fact that a particular line is not part of a major transfer path where the modes can propagate. In the case of the bus frequency, some modes are not observable when the PMU is close to the pivot of the system for each oscillatory mode.

The data is segmented in blocks of 15 minutes, and preprocessed for analysis. For the active power flow signals, the pre-processing is simply done by detrending the data. A sample $15 \mathrm{~min}$. block of the detrended power signal is shown in Fig. $4 \mathrm{a}^{1}$. For bus frequencies, we fist compute the numerical derivative of the measured bus voltage angle, and later apply detrending. This is done to avoid taking into account any prefiltering in the PMU used to obtain bus frequency estimates. To each 15 min. block of pre-processed data we apply the Welch and Yule-Walker methods as described below.

We start by applying the Welch method to the pre-processed data to obtain estimated periodogram spectrums. We use ten minutes of data for the number of FFT points used to calculate the Power Spectrum Density (PSD) estimate. In addition, a Bohman window with $80 \%$ overlap is applied to the data. Fig. $4 \mathrm{~b}$ shows the estimated periodogram for the $15 \mathrm{~min}$. data block in Fig. 4a.

\footnotetext{
${ }^{1}$ For convenience, pre-processed active power flow signals are denoted by $P_{i}$, where $i$ is the line number from where measurements are made.
} 


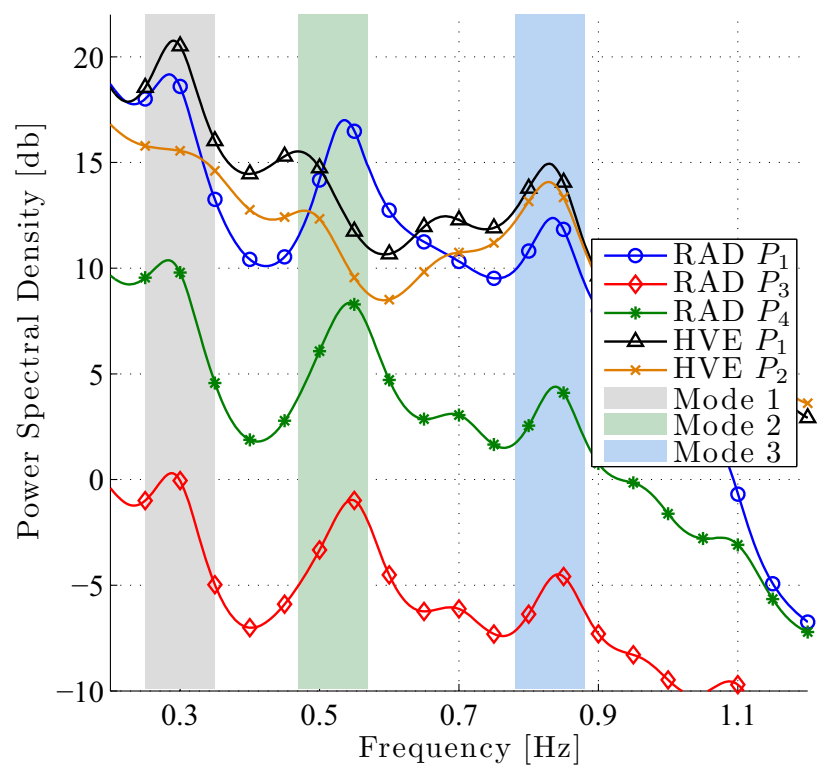

Figure 5. Yule-Walker PDS for all the active power flow signals at Radsted and Hovegård. The $15 \mathrm{~min}$. data blocks for the signals at Radstead are taken during 03-20-2008 and for the signals at Hovegård during 04-15-2007.

Next, we apply the Yule-Walker method to the preprocessed data. The estimated periodograms from Welch's method are used to refine the ARMA model order of the YuleWalker method by comparing the PSD of both methods. As a result, excellent agreement was obtained between the PSDs estimated from each method. In Fig. $4 \mathrm{~b}$ we show the YuleWalker PSD along with the one obtained by Welch's method. Notice the close agreement between both spectrum estimates.

Subsequently, we applied both methods to all remaining signals from Radstead and Hovegård, for 15 min. time-windows similar to the one in Fig. 4a. We show all the estimated PSDs from the YW method in Fig. 5 where the dominant modes have distinctive peaks in the spectral estimate. Note that the estimates for Hovegård are obtained for a different date than those of Radsted. Comparing all the PSDs we have determine three dominant modes for the Nordic system at approximately $0.3,0.5$, and $0.8 \mathrm{~Hz}$, with bounds shown in Fig. 5 .

We repeat the methodology explained above for all the data blocks for a period of $48 \mathrm{hrs}$. As a result, we obtain the contours and surfaces shown in Figs. 6 and 7 for Radsted, and in Fig. 8 for Hovegård. For the $P_{1}$ signal of Radsted we show its Welch contour in Fig. 6a, and its corresponding YW contour in Fig. 7a, observe the close agreement between both contours confirming the existence of the modes and bounds discussed above.

The dynamics of the power system vary over the $48 \mathrm{hrs}$. measurements of Radstead. Note that 03-20-2008 was a "typical day" (in terms of the power system loading), while 0321-2008 was a national holiday with decreased loading. It is important to note that the frequency and damping ratio of the electromechanical modes are influenced by the system loading and configuration of the power grid. For example, Mode $2(0.5$ $\mathrm{Hz}$ ) is present throughout $03-20-2008$, but due to decreased loading on $03-21-2008$ it is not visible during the $32-42 \mathrm{hrs}$. segment (9:00 am - 7:00 pm in local time), which includes regular weekday working hours. It is interesting to observe that as a result of the different loading conditions the frequency of Mode 2 varies during hours 0-32 hrs.

In Fig. 7 we show the Yule-Walker contours for all the active power signals from Radstead, with $P_{1}$ having the highest power densities (corresponding to the highest line loadings). From these contours it's important to note that all spectrum estimates are in agreement with regard to their dominant modes. In Fig. 6b we show a surface plot of the Yule-Walker PSD for the $P_{1}$ signal, which allows to appreciate the mode frequencies of the power system due to the variation of the power system loading.

Similarly for Hovegård, we can compare the Welch contour in Fig. 8a with the YW contour in Fig. 8b which also shows excellent agreement for the estimated PSDs for the first two modes. However, the third mode about $0.8 \mathrm{~Hz}$ it is hardly observable except for 14-16 hrs. and 30-32 hrs. Thus, we could speculate that the $0.8 \mathrm{~Hz}$ mode is local, but eigenanalysis in Section IV suggests that the $0.8 \mathrm{~Hz}$ is an interarea mode. The surface plot for Hovegård in Fig. 8c provides insight on dynamics of the power system during the $48 \mathrm{hr}$. period, note that for 04-15-2007 the modes are not observable during 2 9 hrs. (3:00 am - 10:00 am in local time) which corresponds to a Sunday morning.

\section{B. Mode and Damping Estimates}

The algorithms discussed above produce estimates for different modes. Some of these modes are true system modes, and others are numerical artifacts [4]. Here, we use a modal energy method to determine which modes have the largest energy in the signal in each of the ranges discussed above. An algorithm similar to the one reported in [2], [4] was used for this purpose.

In Fig. 10 we show the frequency and damping estimates for signals $P_{1}, P_{3}$, and $P_{4}$ from Radsted. Similar results were obtained for Hovegård. However, we have omitted the corresponding plots due to space constraints. We plot the damping and frequency estimates for each 15 min. block, and accompany them with their corresponding PSD contour from Welch's method. Observe that as a result of the load variation through the $48 \mathrm{hrs}$. period the estimated frequency and damping ratio for each mode also present changes. The most important characteristic to note is that the the damping estimates are less variable as the system becomes more stressed. In other words, the mode meter algorithm estimates are more reliable as the system stress is increased.

In addition, we have computed the average mode frequencies and damping estimates along with their standard deviations. We provide these statistics in Table I for Radsted, and in Table II for Hovegård. Note that the damping of each mode is different at each substation.

The contour plots from Welch's method accompanying the mode frequency and damping estimates in Fig. 10 provide insight into the damping ratio of each mode. A narrow distinctive frequency band in the contour as those shown for Mode 1 in Figs. 10a and 10d (shown mostly in red between $0.27-0.28 \mathrm{~Hz}$ ) represents a low damping ratio, in this case the average damping ratio is between $2.774-5.1224 \%$ at 
Surface - Yule-Walker PSD - RAD132 P1

Recording Starting at 2008-03-20 00:00:00 (UTC)

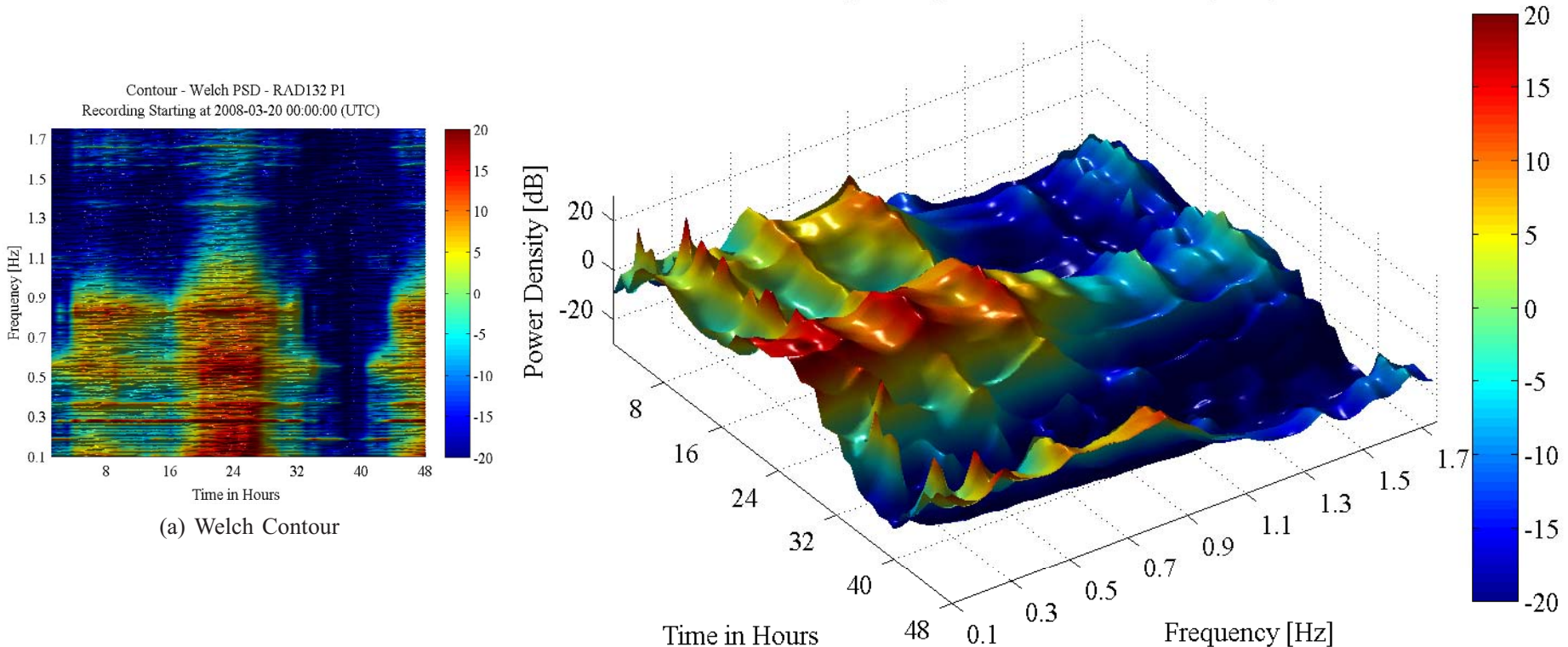

(b) Yule-Walker Surface

Figure 6. Welch and YW PSD Contours, and Yule-Walker Surface for the $P_{1}$ signal from Radsted. The red colors represent maximum values and the blue colors represent minimum values of the power spectrum density [dB]. The time is given in hours in (UTC) starting from 00:00:00 hrs, local time is given in $\mathrm{UTC}+1 \mathrm{hr}$.

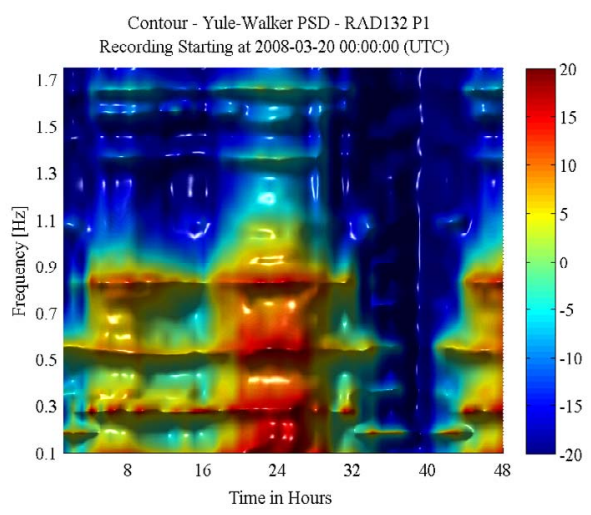

(a) Yule-Walker Contour for $P_{1}$

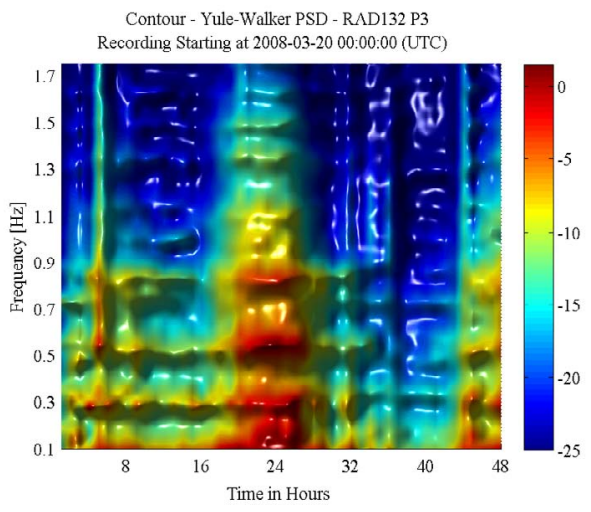

(b) Yule-Walker Contour for $P_{3}$

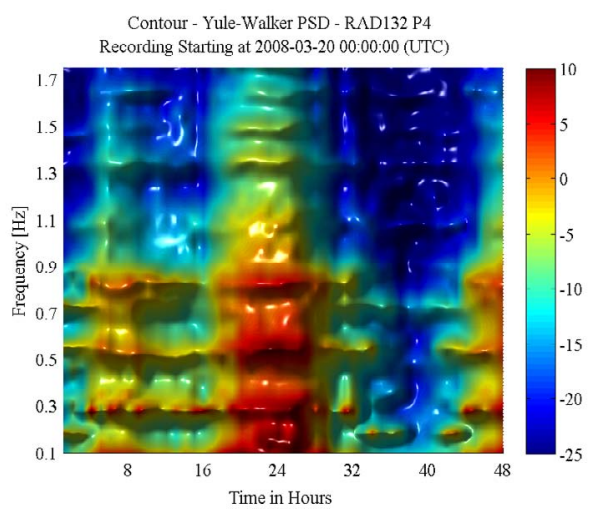

(c) Yule-Walker Contour for $P_{4}$

Figure 7. PSDs Yule-Walker Contours for the $P_{1}, P_{3}, P_{4}$ signals from Radsted. The red colors represent maximum values and the blue colors represent minimum values of the power spectrum density $[\mathrm{dB}]$. The time is given in hours in (UTC) starting from 00:00:00 hrs, local time is given in UTC+1 hr.

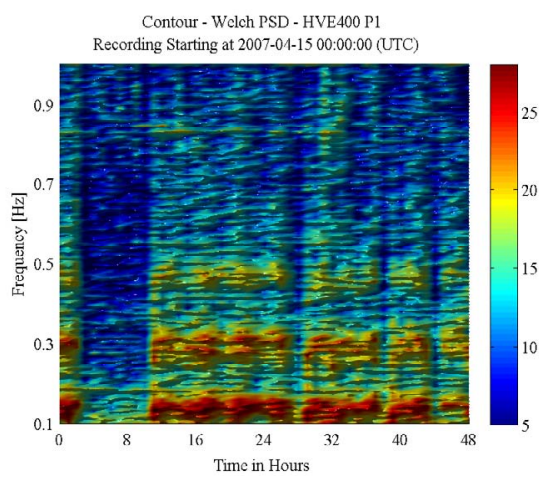

(a) Welch Contour

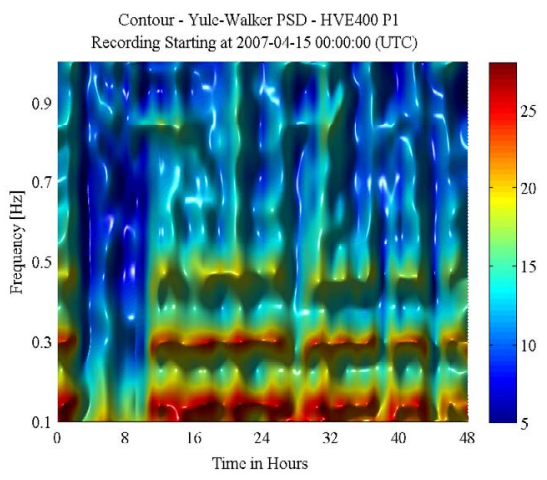

(b) Yule-Walker Contour

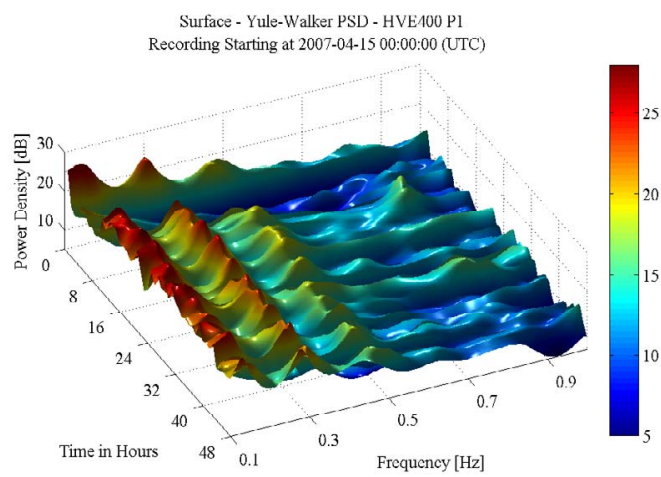

(c) Yule-Walker Surface

Figure 8. PSDs Contours and Surface Plots for the $P_{1}$ signal from Hovegård. The red colors represent maximum values and the blue colors represent minimum values of the power spectrum density [dB]. The time is given in hours in (UTC) starting from 00:00:00 hrs, local time is given in UTC+1 hr. 
Table I

Mode Meter Estimates for Radstead

\begin{tabular}{|c|c|c|c|c|c|}
\hline Mode & SIGNAL & $\bar{f}(\mathrm{~Hz})$ & $\sigma_{f}$ & $\bar{d}(\%)$ & $\sigma_{d}$ \\
\hline \hline \multirow{3}{*}{ Mode 1 } & $P_{1}$ & 0.2784 & 0.0054 & 2.7741 & 3.5577 \\
& $P_{3}$ & 0.2789 & 0.0056 & 5.1224 & 4.1227 \\
& $P_{4}$ & 0.2776 & 0.0052 & 3.1023 & 3.5267 \\
\hline \hline & $P_{1}$ & 0.5462 & 0.0185 & 7.0530 & 4.4575 \\
Mode 2 & $P_{3}$ & 0.5354 & 0.0195 & 10.053 & 4.1227 \\
& $P_{4}$ & 0.5331 & 0.0210 & 7.8131 & 3.7418 \\
\hline \hline & $P_{1}$ & 0.8190 & 0.0221 & 5.1698 & 3.5451 \\
Mode 3 & $P_{3}$ & 0.8224 & 0.0219 & 5.8718 & 3.0722 \\
& $P_{4}$ & 0.8291 & 0.0116 & 4.8269 & 3.5301 \\
& $f_{\tilde{V}}$ & 0.8258 & 0.0352 & 7.3992 & 3.5089 \\
\hline
\end{tabular}

Table II

Mode Meter Estimates for HovegÅ R

\begin{tabular}{|c|c|c|c|c|c|}
\hline Mode & SignAL & $\bar{f}(\mathrm{~Hz})$ & $\sigma_{f}$ & $\bar{d}(\%)$ & $\sigma_{d}$ \\
\hline \hline Mode 1 & $P_{1}$ & 0.2943 & 0.0144 & 14.0157 & 3.2173 \\
\hline \hline \multirow{2}{*}{ Mode 2 } & $P_{1}$ & 0.4926 & 0.0186 & 14.0157 & 3.2173 \\
& $P_{2}$ & 0.5243 & 0.0369 & 13.2147 & 3.7919 \\
\hline \hline \multirow{3}{*}{ Mode 3 } & $P_{1}$ & 0.8238 & 0.0138 & 5.6573 & 3.6906 \\
& $P_{2}$ & 0.8323 & 0.0181 & 6.2275 & 3.6686 \\
& $f_{\tilde{V}}$ & 0.8132 & 0.0295 & 8.0509 & 4.3171 \\
\hline
\end{tabular}

Radstead. Conversely, a broader frequency band will indicate larger damping ratio. For example Mode 2 has a broader frequency band approximately between $0.45 \mathrm{~Hz}$ and $0.6 \mathrm{~Hz}$ as shown Figs. 10b and 10e (shown mostly in yellow and red in $10 \mathrm{~b}$, and in green, yellow, orange, and red in 10e). This band corresponds to a higher damping ratio whose average is between $7.053-10.053 \%$ at Radstead.

In summary, from Tables I and II, the average frequency and damping of the dominant electromechanical modes are between the following ranges:

$\diamond$ Mode 1: $0.2776-0.2943 \mathrm{~Hz}$, with $2.7741-14.0157 \%$ damping

$\diamond$ Mode 2: $0.4926-0.5462 \mathrm{~Hz}$, with $7.0530-14.0157 \%$ damping

$\diamond$ Mode 3: $0.8132-0.8323 \mathrm{~Hz}$, with $4.8269-8.0509 \%$ damping where Mode 1 and Mode 2 are interarea modes.

\section{COMPARISON WITH EIgENANALYSIS INVESTIGATIONS}

In this Section we compare the estimated modes from ambient data analysis to those reported in an extensive eigenanalysis investigation of the Nordic system [13]. Additional results of this eigenanalysis study not available in [13] are also reported.

The modes determined from [13] are obtained for a high loading scenario corresponding to the winter, while the ambient analysis was performed using measurements from Spring '07 and Spring '08. Therefore, differences between the results of both methodologies are expected. Table III shows the interarea modes obtained by each study. The interarea modes estimated in this study are in agreement with the interarea modes reported in [13], it should be noted that the damping estimates from ambient data analysis are more optimistic.

The eigenanalysis study [13] also determined the most important mode shapes for the Nordic System. For Mode 1 $(0.3 \mathrm{~Hz})$ the mode shape shows generators in Finland oscillating against the rest of the Nordic system, including Eastern Denmark. For Mode $2(0.5 \mathrm{~Hz})$ the mode shape shows
Table III

Mode Estimates CoMparison With EIgENANALYSis STUdy FOR THE INTERAREA MODES

\begin{tabular}{|c|c|c|c|c|}
\hline \multirow{2}{*}{ Mode } & \multicolumn{2}{|c|}{ Eigenanalysis } & \multicolumn{2}{c|}{ Ambient Data Analysis } \\
\cline { 2 - 5 } & $f(\mathrm{~Hz})$ & $d(\%)$ & $\bar{f}(\mathrm{~Hz})$ & $\bar{d}(\%)$ \\
\hline Mode 1 & 0.33 & 0.99 & $0.2776-0.2943$ & $2.7741-14.0157$ \\
\hline Mode 2 & 0.48 & 5.05 & $0.4926-0.5462$ & $7.0530-14.0157$ \\
\hline
\end{tabular}

generators in Southern Norway and Finland oscillating against the rest of the system.

The eigenanalysis study [13] focused on determining modes that were most observable and controllable within the Finish grid. Here, we provide additional results from the study in [13] which are relevant to the ambient-data analysis results discussed in this paper, and that where not reported in [13]. During this eigenanalysis study several modes within the frequency range of $0.80-0.88 \mathrm{~Hz}$ were observed. These modes behave either as local modes in Eastern Denmark or interarea modes in Southern Sweden and Denmark, and are therefore outside the scope of the discussion in [13].

Within the frequency range mentioned above, there was a highly observable mode in Eastern Denmark with a frequency of $0.82 \mathrm{~Hz}$, and damping of $11.12 \%$. This $0.82 \mathrm{~Hz}$ mode is an interarea mode between Sweden and Eastern Denmark. A mode shape derived from eigenanalysis of a 3000 - bus model of the Nordic power system is shown in Fig. 9. Presumably, the $0.82 \mathrm{~Hz}$ oscillation observed in this paper is related to the same mode as observed during the study in [13]. The average mode frequency and damping for Mode $3(0.8$ $\mathrm{Hz}$ ) from ambient-data analysis is between $0.8132-0.8323$ $\mathrm{Hz}$ and $5.1698-8.0509 \%$ damping. As mentioned before, the frequency and damping of oscillatory modes are closely related to the operating state of the power system. Therefore, the difference in operating state between the studies should be regarded as one of the influencing factors on the deviation in their results.

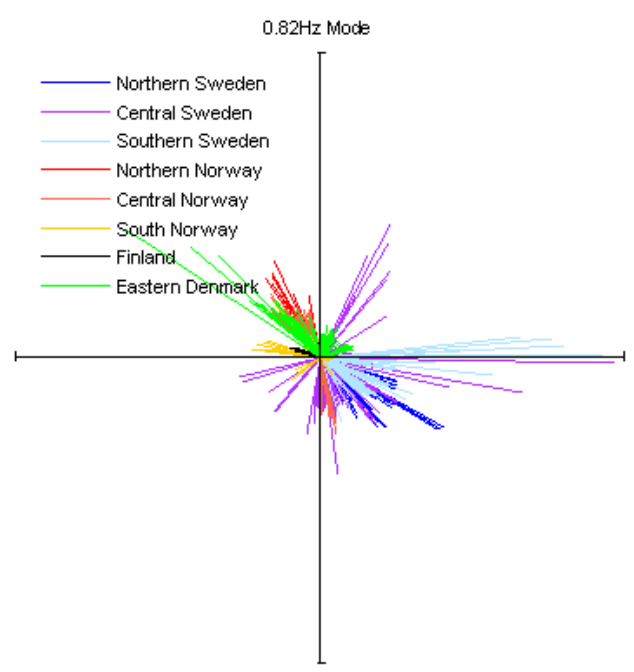

Figure 9. Bus Voltage Angle Mode shape of the $0.82 \mathrm{~Hz}$ mode derived from eigenanalysis of a 3000 bus model of the Nordic power system 
It is also important to mention that the identified interarea modes presented in this study are in close agreement with recent measurement-based studies. In [11], Kalman filtering is used on ring down data identifying interarea modes of 0.3 and $0.5 \mathrm{~Hz}$. In [12] initial spectral analysis resulted in the estimation interarea modes of 0.33 and $0.6 \mathrm{~Hz}$.

\section{CONCLUSIONS}

We have reported the initial results of a collaborative effort to baseline the modes and mode shapes of the Nordic System. This investigation has focused on the application of nonparametric and parametric methods for mode frequency and damping estimation. We have obtained interarea modes that are in close agreement with published results on eigenvalue analysis of planning models. The discovery of a $0.8 \mathrm{~Hz}$ mode in Eastern Denmark is also reported, eigenanalysis suggests that this mode is of interarea nature involving the swing of generators in Sweden and Eastern Denmark.

Because the data sets used in this study were not recorded during the same dates it was not possible to compute the CSD, determine coherency, and obtain mode shapes. Further work will be focused on determining the mode shape and coherency characteristics of the network for the identified interarea modes using simultaneous phasor measurements from all available PMUs in Eastern Denmark. We also plan on confirming our ambient data analysis using ring down analysis (such as the ERA [14] and Prony [15] algorithms) on a major system disturbance that took place during 2007 for which phasor measurements are available.

This work should be viewed as the starting point of a research effort aiming to fully characterize the electromechanical modes and mode shapes in the Nordic system. Hence, the authors are looking for universities and utilities interested to join this effort to continue research and to provide phasor measurements deployed across the Nordic system.

\section{ACKNOWLEDGMENT}

L. Vanfretti, J.H. Chow, D. Trudnowski, and J. W. Pierre would like to acknowledge the support of the RPI Power System Research Consortium Industry Members: AEP, FirstEnergy, NE-ISO, NYISO, and PJM, and the NSF through grant ECS-0622119.

\section{REFERENCES}

[1] A. Hauer, D. Trudnowski, and J. DeSteese, "A Perspective on WAMS Analysis Tools for Tracking of Oscillatory Dynamics," IEEE Power Engineering Society General Meeting, 2007., pp. 1-10, June 2007.

[2] D. Trudnowski, J. Pierre, N. Zhou, J. Hauer, and M. Parashar, "Performance of Three Mode-Meter Block-Processing Algorithms for $\mathrm{Au}-$ tomated Dynamic Stability Assessment," IEEE Transactions on Power Systems, vol. 23, no. 2, pp. 680-690, May 2008.

[3] L. Vanfretti, T. M. L. Assis, J. H. Chow, L. Dosiek, J. W. Pierre, D. Trudnowski, and Y. Liu, "Data Analysis of the 2/26/08 Florida Blackout," NASPI Work Group Meeting, Sacramento, CA, June 4, 2009, available online:http://www.naspi.org/meetings/workgroup/workgroup.stm.

[4] D. Trudnowski and J.W. Pierre, in Inter-area Oscillations in Power Systems: A Nonlinear and Nonstationary Perspective, ser. Power Electronics and Power Systems, A. R. Messina, Ed. Springer, 2009, ch. Signal Processing Methods for Estimating Small-Signal Dynamic Properties from Measured Responses, pp. 1-36.

[5] F. Tuffner and J. Pierre, "Electromechanical Modal Behavior During a 48 Hour Interval Using Nonparametric Methods," Proceedings of the North American Power Symposium (NAPS), September 2006.
[6] J. Pierre and R. F. Kubichek, "Spectral Analysis: Analyzing a Signal Spectrum," Tektronix Application Note, 2002, Available online: http://www.tek.com/Measurement/App_Notes/55_15429/eng/.

[7] J. Proakis and D. Manolakis, Digital Signal Processing Principles, Algorithms, and Applications, 4th ed. Prentice Hall, 2007.

[8] J. Pierre, D. Trudnowski, and M. Donnelly, "Initial Results in Electromechanical Mode Identification from Ambient Data," IEEE Transactions on Power Systems, vol. 12, no. 3, pp. 1245-1251, Aug. 1997.

[9] D. Trudnowski, "Estimating Electromechanical Mode Shape From Synchrophasor Measurements," IEEE Transactions on Power Systems, vol. 23, no. 3, pp. 1188-1195, Aug. 2008.

[10] D. Trudnowski, J. Hauer, J. Pierre, W. Litzenberger, and D. Maratukulam, "Using the coherency function to detect large-scale dynamic system modal observability," Proceedings of the 1999 American Control Conference, vol. 4, pp. 2886-2890, 1999.

[11] K. Uhlen, L. Warland, J. Gjerde, O. Breidablik, M. Uusitalo, A. Leirbukt, and P. Korba, "Monitoring amplitude, frequency and damping of power system oscillations with PMU measurements," IEEE Power and Energy Society General Meeting - Conversion and Delivery of Electrical Energy in the 21st Century, 2008, pp. 1-7, July 2008.

[12] M. Hemmingsson, O. Samujelsson, K. Pedersen, and A. Nielsen, "Estimation of electro-mechanical mode parameters using frequency measurements," IEEE Power Engineering Society Winter Meeting, 2001, vol. 3, pp. 1172-1177 vol.3, 2001.

[13] K. Uhlen, S. Elenius, I. Norheim, J. Jyrinsalo, J. Elovaara, and E. Lakervi, "Application of linear analysis for stability improvements in the Nordic power transmission system," IEEE Power Engineering Society General Meeting, 2003, vol. 4, pp. -2103 Vol. 4, July 2003.

[14] J. Sanchez-Gasca and J. Chow, "Performance comparison of three identification methods for the analysis of electromechanical oscillations," IEEE Transactions on Power Systems, vol. 14, no. 3, pp. 995-1002, Aug. 1999.

[15] J. F. Hauer, C. J. Demeure, and L. L. Scharf, "Initial results in Prony analysis of power system response signals," IEEE Transactions on Power Systems, vol. 5, no. 1, pp. 80-89, Feb. 1990.

Luigi Vanfretti (Sudent Member '03) is a Post-Doctoral Research Associate at the Electrical, Computer, and Systems Engineering Department at Rensselaer Polytechnic Institute, from where he obtained his PhD in 2009, and MS in 2007, both in Electric Power Engineering. He received the Electrical Engineering degree from Universidad de San Carlos de Guatemala in Spring 2005. He was a visiting researcher at the Department of Electronics and Electrical Engineering of The University of Glasgow, Scotland, during Fall 2005. His research interests are modeling, dynamics, stability and control of power systems; applications of PMU data, and open source software for power system analysis.

Rodrigo García-Valle was born in México. He received the electrical engineering degree from the National Polytechnic Institute of México, in 2001, the M.Sc. degree from CINVESTAV, Guadalajara, México, in 2003 and obtained his Ph.D. degree from the University of Glasgow, U.K., in 2007. In 2008, he was granted with the Hans Christened Ørsted Award at the Centre for Electric Technology (CET) by the Technical University of Denmark to carry out postdoctoral research activities. Since 2009 he holds the position as Assistant Professor. His research interests are dynamics, stability and control of electric power systems; artificial intelligence techniques; renewable energy integration; and modeling and simulation of FACTS and custom power controllers. He is an IEEE, IET and CIGRE member.

Kjetil Uhlen (M'95) was born in 1961. He received the Sivilingeniør degree from the Norwegian Institute of Technology in 1986 and a Ph.D. degree in control engineering from the same institute in 1994. Since 1987 he has worked at SINTEF Energy Research in Trondheim, presently as team leader and senior research scientist.

Emil Johansson was born in Sweden 1975 and received his M.Sc. in electrical engineering at the Swedish Royal Institute of Technology (KTH), Stockholm, in 2002. Between 2001 and 2007 he worked for ABB with HVDC and harmonic filtering. He is currently with SINTEF Energy Research in Trondheim as a research scientist.

Daniel J. Trudnowski (SM'99) received the B.S. degree in engineering science from Montana Tech, Butte, in 1986 and the M.S. and Ph.D. degrees in electrical engineering from Montana State University, Bozeman, in 1988 and 1991, respectively. From 1991 to 1995, he was with Pacific Northwest National Laboratory, where he was a research engineer working on control system and power system dynamic problems. In 1996, he joined Montana Tech, where he is currently Professor and Head of the Electrical Engineering Program. 


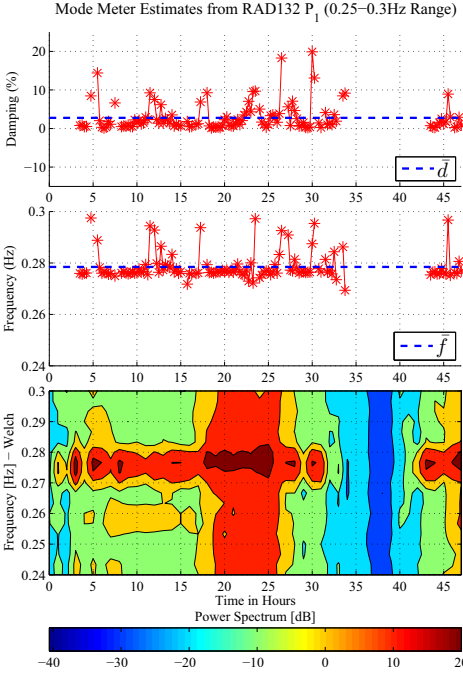

(a) Mode 1 from $P_{1}$

Mode Meter Estimates from RAD132 $\mathrm{P}_{3}(0.22-0.3 \mathrm{~Hz}$ Range $)$

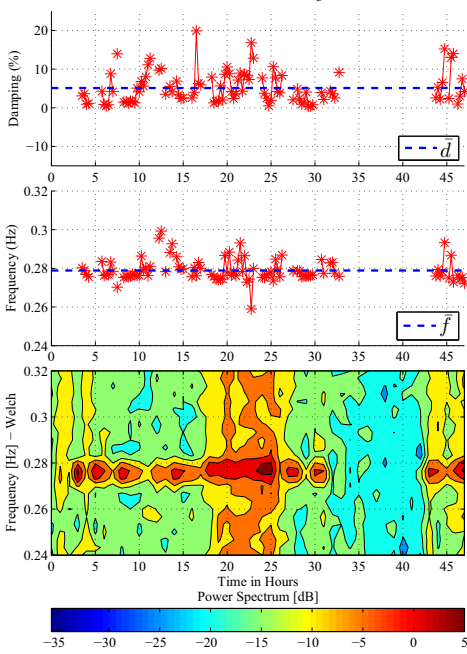

(d) Mode 1 from $P_{3}$

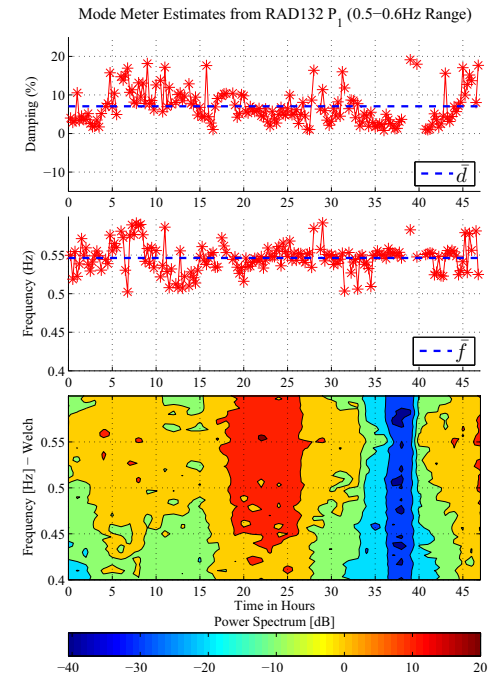

(b) Mode 2 from $P_{1}$

Mode Meter Estimates from RAD132 $\mathrm{P}_{3}(0.495-0.6 \mathrm{~Hz}$ Range $)$
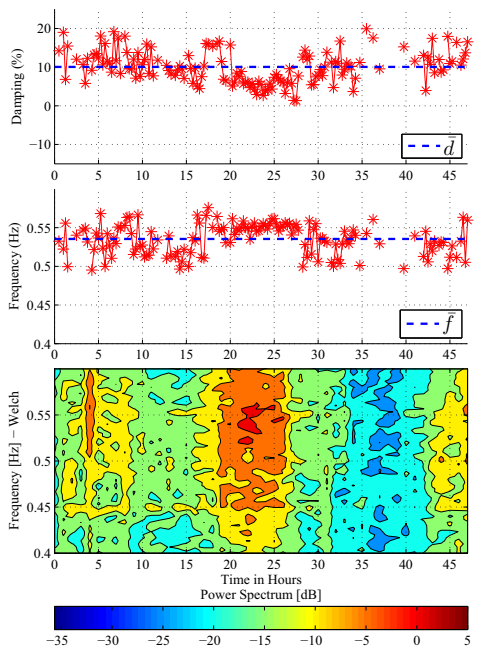

(e) Mode 2 from $P_{3}$

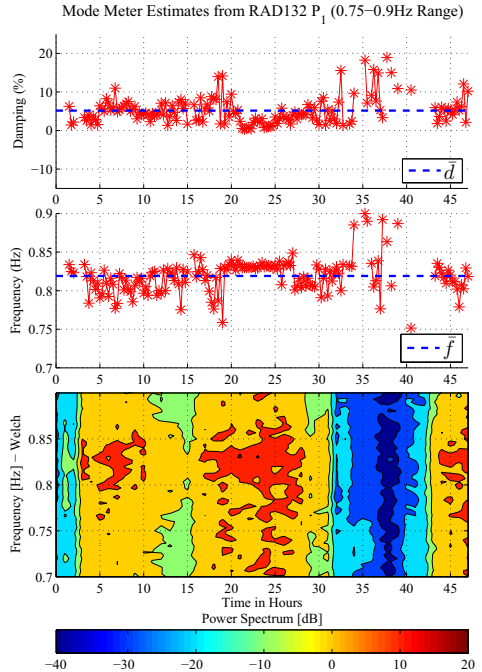

(c) Mode 3 from $P_{1}$

Mode Meter Estimates from RAD132 $\mathrm{P}_{3}(0.775-0.9 \mathrm{~Hz}$ Range)

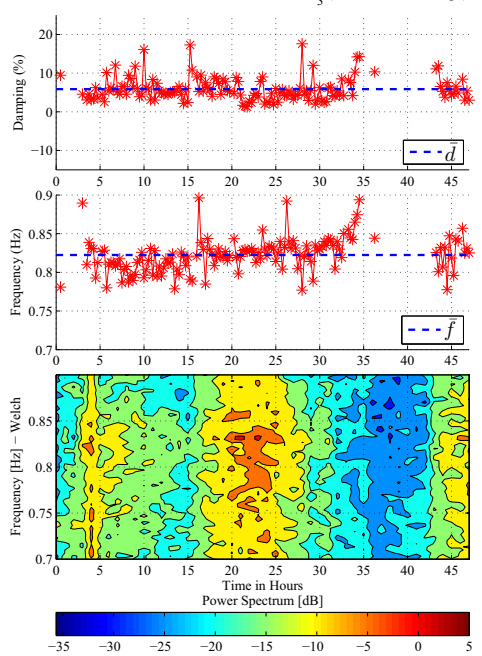

(f) Mode 3 from $P_{3}$

Figure 10. Mode frequency and damping estimates obtained using the mode meter algorithm obtained from Rastead's $R, P_{3}, P_{4}$ signals. In the contours the red colors represent maximum values and the blue colors represent minimum values of the power spectrum density [dB]. The time is given in hours in (UTC) starting from 00:00:00 hrs, local time is given in UTC $+1 \mathrm{hr}$.

John Pierre (SM'99) received the B.S. degree in electrical engineering with a minor in economics from Montana State University, Bozeman, in 1986 and the M.S. degree in electrical engineering with a minor in statistics and the Ph.D. degree in electrical engineering from the University of Minnesota, Twin Cities, in 1989 and 1991, respectively. He worked as an electrical design engineer at Tektronix before attending the University of Minnesota. Since 1992, he has been a Professor at the University of Wyoming (UW), Laramie, in the Electrical and Computer Engineering Department. He served as Interim Department Head from 2003 to 2004. For part of his 20072008 sabbatical, he worked at Pacific Northwest National Laboratory, Richland, WA, and Montana Tech, Butte. His research interests include statistical signal processing applied to power systems as well as DSP education.

Joe H. Chow (F'92) received his MS and PhD degrees from the University of Illinois, Urbana-Champaign. After working in the General Electric Power System business in Schenectady, he joined Rensselaer Polytechnic Institute in 1987. He is a professor of Electrical, Computer, and Systems Engineering and the Associate Dean of Engineering for Research and Graduate Programs His research interests include multivariable control, power system dynamics and control, voltage-sourced converter-based FACTS Controllers, and synchronized phasor data.
Olof Samuelsson (M'98) is associate professor at Department of Measurement Technology and Industrial Electrical Engineering, Lund University, where he also receieved his $\mathrm{PhD}$. His main research interests are stability, control and protection of generation (large and small-scale) and the power system, which includes network integration of distributed generation, island operation of distribution networks and control and also PMU monitoring and damping of transmission systems.

Jacob Østergaard (M'95) is Professor and Head of the Centre for Electric Technology, in the Department of Electrical Engineering, Technical University of Denmark. His research interests include integration of renewable energy, control architecture for future power system, and demand side. Professor Østergaard is serving in several professional organizations including the EU SmartGrids advisory council.

Kenneth E. Martin (F'08) received the B.S.E.E. degree from Colorado State University, Fort Collins, and the M.A. degree from the University of Washington, Seattle. He was a Principal Engineer at the Bonneville Power Administration until 2008 when he joined the Electric Power Group (EPG). At EPG he developed the ePDC for phasor measurement systems and supports design and implementation of phasor measurement WAMS systems worldwide. He has authored or coauthored over 50 technical papers in his specialty areas. Mr. Martin is a member of the Power System Relay Committee and the Relay Communications Subcommittee. He chairs the Synchrophasor Standard working group and the IEC 61850 synchrophasor task team, and is a member of other PSRC and NASPI working groups. He is a registered Professional Engineer in the States of Washington and Oregon. 